\title{
The Chiral Condensate of One-Flavor QCD and the Dirac Spectrum at $\boldsymbol{\theta}=\mathbf{0}$
}

\author{
Jacobus Verbaarschot* \\ Stony Brook University \\ E-mail: jacobus.verbaarschotestonybrook • edu

\section{Tilo Wettig} \\ University of Regensburg \\ E-mail: tilo.wettig@ur.de
}

In a sector of fixed topological charge, the chiral condensate has a discontinuity given by the Banks-Casher formula also in the case of one-flavor QCD. However, at fixed $\theta$-angle, the chiral condensate remains constant when the quark mass crosses zero. To reconcile these contradictory observations, we have evaluated the spectral density of one-flavor QCD at $\theta=0$. For negative quark mass, it becomes a strongly oscillating function with a period that scales as the inverse space-time volume and an amplitude that increases exponentially with the space-time volume. As we have learned from QCD at nonzero chemical potential, if this is the case, an alternative to the Banks-Casher formula applies, and as we will demonstrate in this talk, for one-flavor QCD this results in a continuous chiral condensate. A special role is played by the topological zero modes which have to be taken into account exactly in order to get a finite chiral condensate in the thermodynamic limit.

The 32nd International Symposium on Lattice Field Theory,

23-28 June, 2014

Columbia University New York, $N Y$

\footnotetext{
*Speaker.
} 

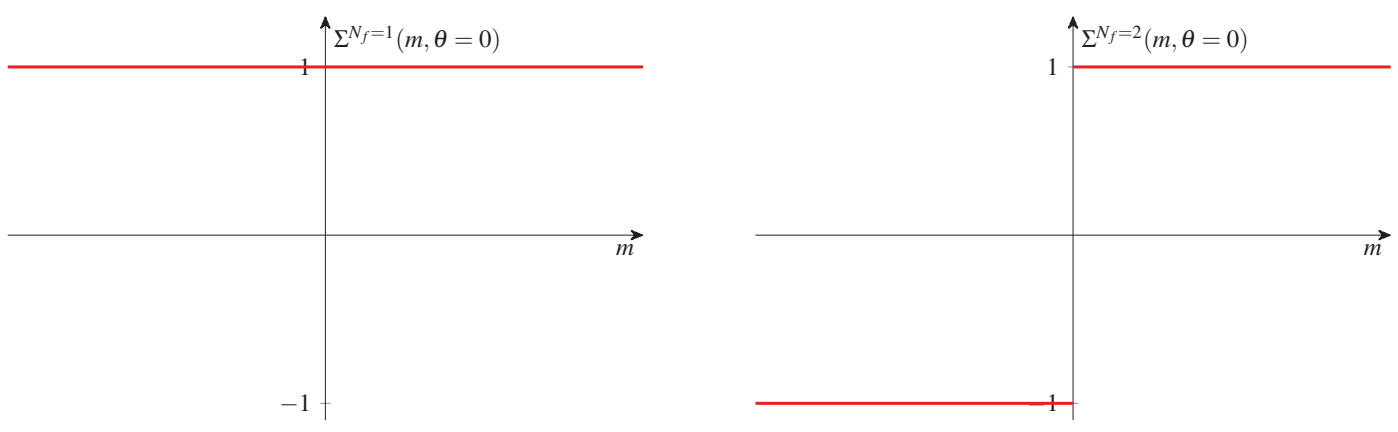

Figure 1: Behavior of the chiral condensate at fixed $\theta$-angle for $N_{f}=1$ (left) and $N_{f}=2$ (right).

\section{Introduction}

In one-flavor QCD, the chiral condensate is continuous as a function of the quark mass despite the fact that the eigenvalues of the Dirac operator become dense on the imaginary axis in the thermodynamic limit. This contradicts a naive application of the Banks-Casher formula [1]. As was already observed before $[2,3]$, the resolution of this paradox is that for negative quark mass the partition function is not positive definite which, as we will see today, results in a strongly oscillating spectral density which cancels the discontinuity of the sign-quenched theory (i.e., the theory in which the determinant of the Dirac operator is replaced by its absolute value). This mechanism is reminiscent of the solution to the Silver Blaze problem [4] for QCD at nonzero chemical potential [5]. In that case the chiral condensate of the phase-quenched theory does not have a discontinuity, but the phase of the fermion determinant results in a strongly oscillating spectral density which cancels the contribution from the phase-quenched theory and gives rise to a discontinuity in the chiral condensate. The original motivation of the present work was to improve our understanding of this relationship between the spectral density and the chiral condensate.

In this talk we explain how the chiral condensate of one-flavor QCD can remain constant when the quark mass traverses zero, while for each gauge-field configuration the eigenvalues of the Dirac operator are spaced on average as $1 / V$, where $V$ is the space-time volume. We do this in the microscopic domain of QCD where all expressions can be worked out analytically.

\section{Formulation of the Problem}

The mass dependence of the chiral condensate of the one-flavor theory is drastically different from the two-flavor theory. In the two-flavor theory, the chiral condensate has a discontinuity at $m=0$ while for $N_{f}=1$ it remains constant, see Fig. 1 . The chiral condensate is defined as

$$
\Sigma(m)=-\langle\bar{q} q\rangle=\frac{1}{V} \frac{d}{d m} \log Z(m),
$$

where $V$ is taken to be large and the quark mass satisfies $\Delta \lambda \ll m \ll \Lambda_{\mathrm{QCD}}$ with $\Delta \lambda$ the average spacing of the eigenvalues. The discontinuity of $\Sigma(m)$ in the thermodynamic limit is a consequence of the spontaneous breaking of chiral symmetry, which does not happen for one-flavor QCD.

It has been well established that at fixed topological charge the low-lying eigenvalues of oneflavor QCD are described by chiral random matrix theory $[7,8,9,10]$. As an example, we show 


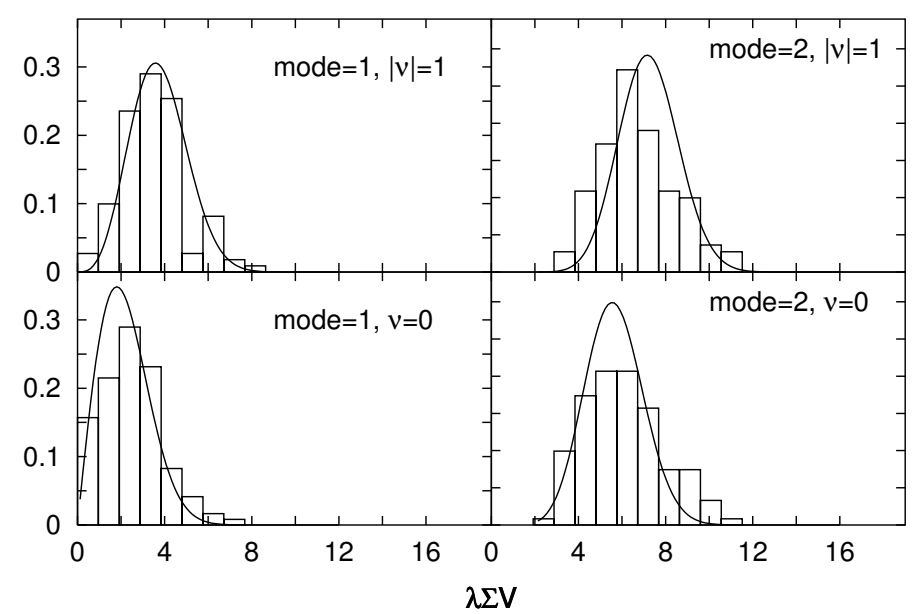

Figure 2: Distribution of the lowest two Dirac eigenvalues for QCD with one flavor compared to the results from chiral random matrix theory (solid curves). Taken from [6].

in Fig. 2 distributions of individual eigenvalues obtained from lattice studies [6]. We emphasize that the smallest Dirac eigenvalues of one-flavor QCD behave in exactly the same way as the Dirac spectrum of QCD and QCD-like theories with spontaneously broken chiral symmetry. If we know the spectral density we can calculate the chiral condensate according to

$$
\Sigma(m)=\left\langle\frac{1}{V} \sum_{k} \frac{1}{i \lambda_{k}+m}\right\rangle=\frac{1}{V} \int d \lambda \frac{\rho(\lambda, m)}{i \lambda+m},
$$

which is valid both at fixed $v$ and at fixed $\theta$. The Banks-Casher formula [1] is obtained in the limit $m \rightarrow 0$,

$$
\Sigma(m \rightarrow 0)=\operatorname{sign}(m) \lim _{\lambda \rightarrow 0} \lim _{m \rightarrow 0} \lim _{V \rightarrow \infty} \frac{\pi}{V}\langle\rho(\lambda, m)\rangle .
$$

A continuous chiral condensate could be obtained if $\rho\left(\lambda, 0_{-}\right)=-\rho\left(\lambda, 0_{+}\right)$for $\lambda \rightarrow 0$ or if $\rho(\lambda, m)=0$ for small $\lambda$ and $m \rightarrow 0[13,14]$, but we will show that what is going on is more subtle. In Fig. 3 (left) we show the mass dependence of the chiral condensate for $v=2$ as a function of $m V$. For $m<0$, the negative parts of $\Sigma_{v}(m)$ in the sum

$$
\Sigma(m, \theta=0)=\frac{\sum_{v=-\infty}^{\infty} Z_{v}(m) \Sigma_{v}(m)}{\sum_{v=-\infty}^{\infty} Z_{v}(m)}
$$

where $Z_{v}(m)=I_{v}(m V)$, should average to a positive number and reproduce the mass dependence shown in the right part of Fig. 3. This is actually possible because

$$
Z_{v}(-|m|) \sim(-1)^{v}|m|^{v}
$$

for small $|m|$. The condensate $\Sigma(m, \theta=0)$ follows from the spectral density at $\theta=0$,

$$
\rho(\lambda, m, \theta=0)=\frac{\sum_{v=-\infty}^{\infty} Z_{v}(m) \rho_{v}(\lambda, m)}{\sum_{v=-\infty}^{\infty} Z_{v}(m)} .
$$



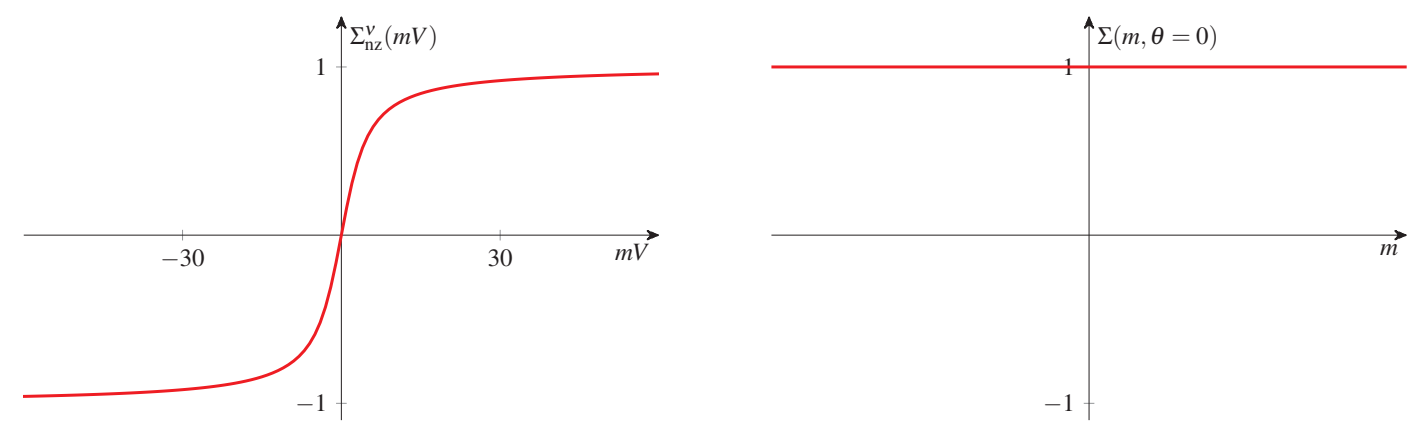

Figure 3: Mass dependence of the chiral condensate due to the nonzero modes for $v=2$ (left) and mass dependence of the chiral condensate at $\theta=0$ (right). Note that $\Sigma_{\mathrm{nz}}^{v}(m V)=\Sigma_{v}(m V)-\frac{|v|}{m V}$. If plotted as a function of $m$, the left curve becomes a step function for $V \rightarrow \infty$.

This spectral density has been evaluated numerically in the $\varepsilon$-domain of QCD [11, 3], but to reconcile the behavior of the chiral condensate at fixed $v$ with the mass independence of the chiral condensate at $\theta=0$ we need explicit analytical expressions for the spectral density. As was discussed in the introduction, we have learned from QCD at nonzero chemical potential that when the eigenvalue density is not positive definite (due to the fermion determinant), the OSV mechanism [5] replaces the Banks-Casher formula. Let us see how this works for $N_{f}=1$.

In Fig. 4 we compare the mass dependence of the chiral condensate in the sign-quenched oneflavor theory (left) with the mass dependence of the chiral condensate in the full theory (right), both at $\theta=0$. These figures imply that the phase factor $(-1)^{v}$ should give a correction to the spectral density (including the zero-mode part) that contributes to the chiral condensate as $\Sigma_{\text {osc }}(m)+$ $\Sigma_{\delta \mathrm{zm}}(m)=2 \theta(-m)$ (see (3.2) for the notation) so that the total chiral condensate remains constant as a function of $m$, see Fig. 5. The oscillating part of the spectral density thus has to satisfy

$$
2 \theta(-m)=\int d \lambda \frac{\rho_{\mathrm{osc}}(\lambda, m)+\rho_{\delta \mathrm{zm}}(\lambda, m)}{i \lambda+m} .
$$

One hint for the solution to this equation comes from the Fourier decomposition of the $\theta$-function,

$$
\theta(m)=\frac{1}{2 \pi i} \int_{-\infty}^{\infty} d \tau \frac{e^{i \tau+m}}{\tau-i m}
$$
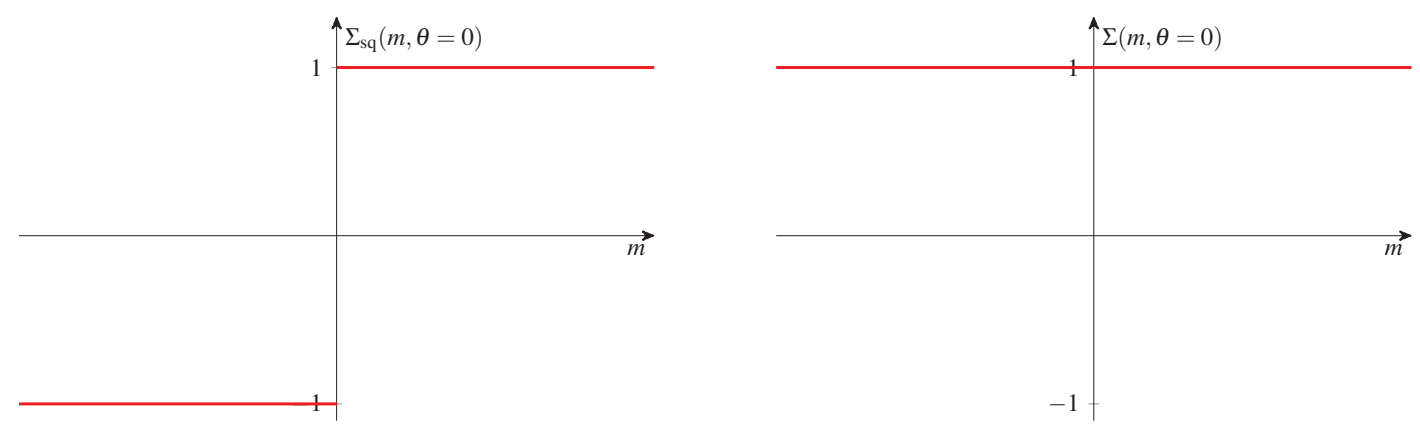

Figure 4: Behavior of the chiral condensate at $\theta=0$ due to a line of eigenvalues for the sign-quenched theory (left) and for the full one-flavor theory (right). 

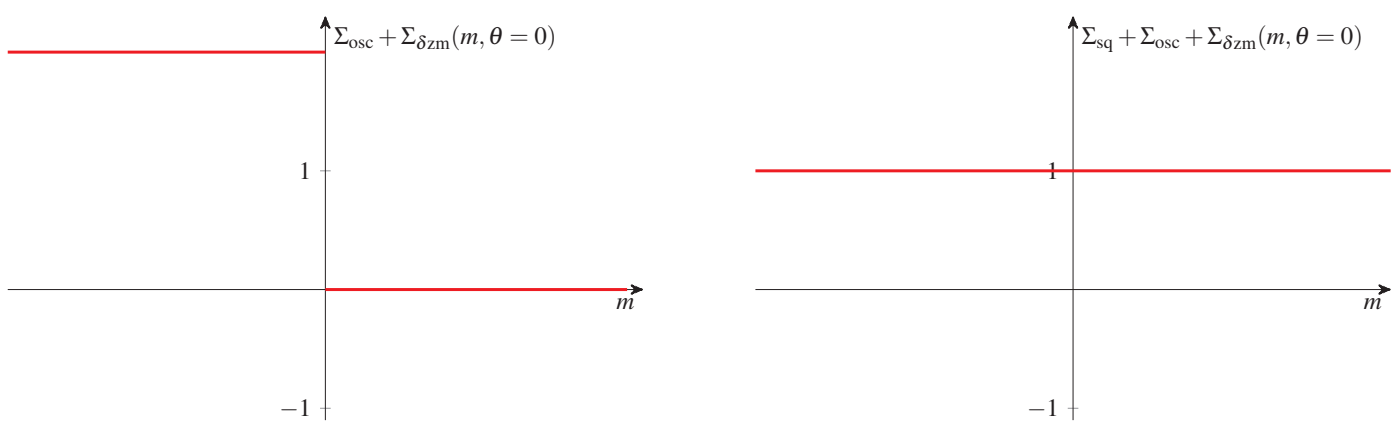

Figure 5: Mass dependence of the chiral condensate due to the oscillating part of the spectral density and the zero modes (left). When the contribution of the sign-quenched part of the spectral density is added the condensate is constant (right).

As an example, it is a simple exercise to show that the spectral density

$$
\rho_{\mathrm{ex}}(\lambda, m)=-\frac{1}{\pi}\left(e^{i \lambda V-m V}+e^{-i \lambda V-m V}\right)
$$

(which is even in $\lambda$ ) results in a mass dependence of the chiral condensate given by

$$
-\frac{1}{\pi} \int_{-\infty}^{\infty} d \lambda \frac{1}{i \lambda+m}\left(e^{i \lambda V-m V}+e^{-i \lambda V-m V}\right)=2 \theta(-m)-2 \theta(m) e^{-2 m V} .
$$

In the thermodynamic limit this results in a continuous chiral condensate. However, for $N_{f}=1$ we have a stronger requirement, namely that the chiral condensate is already mass independent for $m V \sim \mathscr{O}(1)$, which is not the case in the example above. Let us therefore calculate the spectral density for one-flavor QCD.

\section{Spectral Density and Chiral Condensate for One-Flavor QCD}

In the microscopic domain of QCD, where the quark mass and the Dirac eigenvalues scale as

$$
m \sim \frac{1}{V}, \quad \lambda \sim \frac{1}{V},
$$

the spectral density at fixed $v$ is known analytically [9]. The one-flavor spectral density at $\theta=0$ can be decomposed in different ways,

$$
\rho(\lambda, m, \theta=0)=\left\{\begin{array}{l}
\rho_{\mathrm{zm}}(\lambda, m)+\rho_{\mathrm{nz}}(\lambda, m), \\
\rho_{\mathrm{sq}}(\lambda, m)+\rho_{\mathrm{osc}}(\lambda, m)+\rho_{\delta \mathrm{zm}}(\lambda, m),
\end{array}\right.
$$

where the subscripts stand for zero modes, nonzero modes, sign-quenched, oscillating, and the difference in the zero-mode density between full and sign-quenched theory. We have

$$
\begin{aligned}
\rho_{\mathrm{sq}}(\lambda, m) & =\rho(\lambda,|m|), \\
\rho_{\mathrm{osc}}(\lambda, m) & =\rho_{\mathrm{nz}}(\lambda, m)-\rho_{\mathrm{nz}}(\lambda,|m|), \\
\rho_{\delta \mathrm{zm}}(\lambda, m) & =\rho_{\mathrm{zm}}(\lambda, m)-\rho_{\mathrm{zm}}(\lambda,|m|) .
\end{aligned}
$$



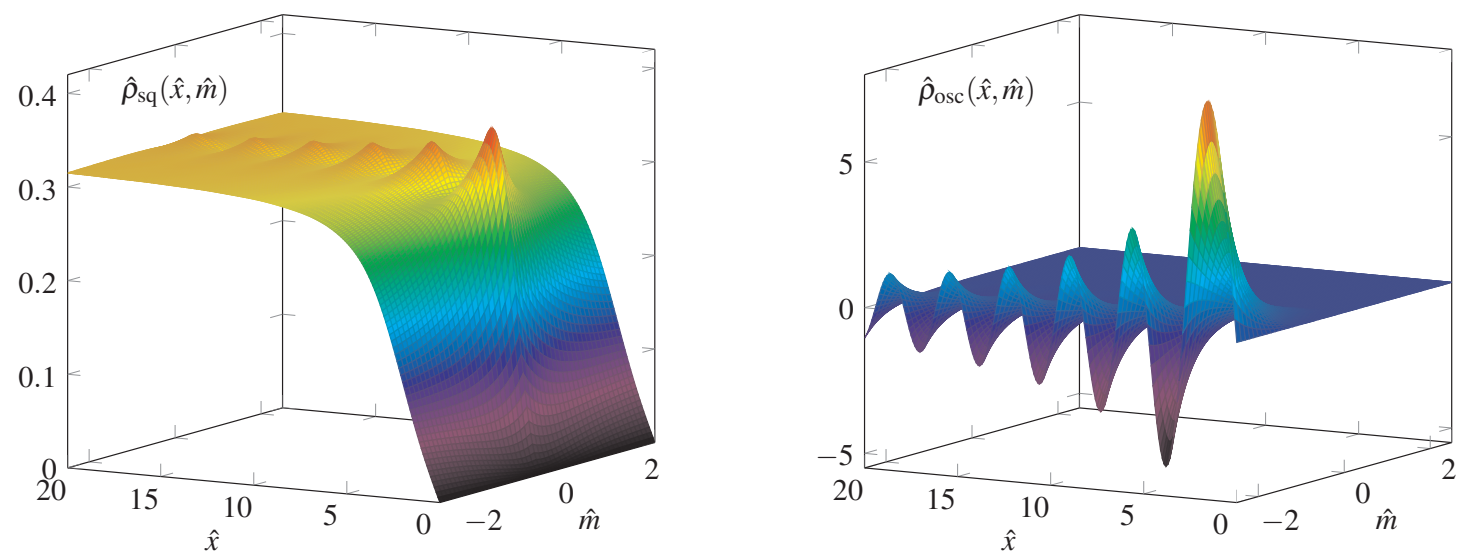

Figure 6: The sign-quenched (left) and oscillating part (right) of the spectral density at $\theta=0$ as a function of $\hat{x}$ and the quark mass $\hat{m}$.

Note that in Ref. [12] we used a different decomposition with $\hat{\rho}_{\mathrm{nz}}(\lambda, m)=\hat{\rho}_{\mathrm{q}}(\lambda, m)+\hat{\rho}_{\mathrm{d}}(\lambda, m)$, but the decomposition (3.2) is more intuitive. The sum (2.6) over $v$ for each of the terms can be evaluated using identities for sums of products of Bessel functions (see [12]),

$$
\begin{aligned}
& \hat{\rho}_{\mathrm{zm}}(\hat{x}, \hat{m})=e^{-\hat{m}} \sum_{V}|v| I_{v}(\hat{m}) \delta(\hat{x})=\hat{m} e^{-\hat{m}}\left[I_{0}(\hat{m})+I_{1}(\hat{m})\right] \delta(\hat{x}), \\
& \hat{\rho}_{\mathrm{nz}}(\hat{x}, \hat{m})=\frac{1}{\pi} \int_{0}^{1} \frac{e^{-2 \hat{m} t^{2}} d t}{\sqrt{1-t^{2}}}\left[\frac{J_{1}(2|\hat{x}| t)}{t}-\frac{2|\hat{x}|}{\hat{x}^{2}+\hat{m}^{2}}\left[\hat{x} t J_{1}(2 \hat{x} t)+\hat{m}\left(1-2 t^{2}\right) J_{0}(2 \hat{x} t)\right]\right]
\end{aligned}
$$

with $\hat{x}=\lambda V$ and $\hat{m}=m V$. In Fig. 6 we show three-dimensional plots of $\hat{\rho}_{\mathrm{sq}}(\hat{x}, \hat{m})$ (left) and $\hat{\rho}_{\text {osc }}(\hat{x}, \hat{m})$ (right). For $m>0$ we trivially have $\hat{\rho}_{\text {osc }}=0$, but for $m<0$ we observe strong oscillations.

The chiral condensate can be obtained by integration over the spectral density, see Eq. (2.2). For each of the contributions to the spectral density this results in an expression given by a simple one-dimensional integral involving modified Bessel functions. Writing $\Sigma(m)=\Sigma_{\mathrm{sq}}(m)+\Sigma_{\mathrm{osc}}(m)+$ $\Sigma_{\delta \mathrm{zm}}(m)$, the explicit expressions for these three terms follow immediately from the expressions for $\Sigma^{\mathrm{zm}}, \Sigma^{q}$, and $\Sigma^{d}$ in [12]. We observe that $\Sigma_{\mathrm{sq}}(m)$ is given by Fig. 4 (left), while for $m<0$, $\Sigma_{\text {osc }}(m)$ diverges in the thermodynamic limit as

$$
-\frac{e^{2|m V|}}{\sqrt{8 \pi|m V|^{3}}} .
$$

This divergence is canceled by a similar contribution from the difference in the zero-mode density [3], see Fig. 7. Using the exact expressions we can show that the cancellations take place to all orders [12] and that we obtain Fig. 5 (left) as required. This can be done analytically by expressing the integrand in terms of total derivatives [12].

\section{Conclusions}

We have obtained exact analytical expressions for the spectral density of one-flavor QCD at $\theta=0$. For negative quark mass the spectral density becomes strongly oscillating, and after canceling a divergent contribution from the zero modes we find a chiral condensate that does not 


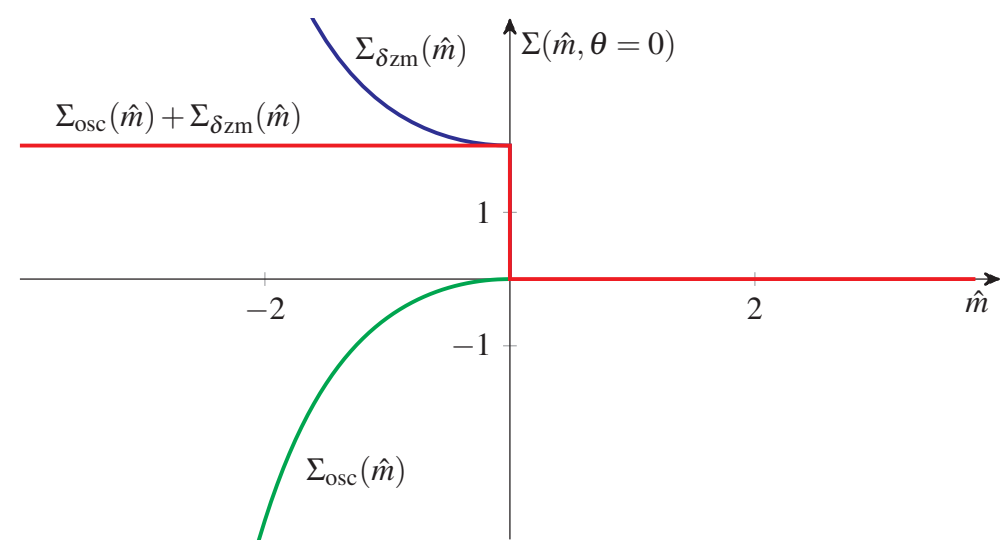

Figure 7: The exponentially large contribution of the difference in the zero-modes density (blue) is canceled by the contribution of the oscillating part of the spectral density (green). The sum of the two contributions is given by the red curve. Note that the curves are plotted as a function of $\hat{m}=m V$.

depend on the quark mass. That a strongly oscillating contribution to the spectral density can result in a discontinuity of the chiral condensate was first observed for QCD at nonzero chemical potential. What is different for one-flavor QCD is that the discontinuity of the sign-quenched condensate is canceled by a discontinuity due to the oscillatory part of the spectral density and the difference in the zero-mode density. An additional subtlety is that the oscillatory part cancels a divergent contribution due to the zero modes.

\section{References}

[1] T. Banks and A. Casher, Nucl. Phys. B169 (1980) 103.

[2] H. Leutwyler and A. V. Smilga, Phys. Rev. D46 (1992) 5607.

[3] T. Kanazawa, T. Wettig, and N. Yamamoto, JHEP 12 (2011) 007 [arXiv:1110.5858].

[4] T. D. Cohen, Phys. Rev. Lett. 91 (2003) 222001 [hep-ph/0307089].

[5] J. C. Osborn, K. Splittorff, and J. J. M. Verbaarschot, Phys. Rev. Lett. 94 (2005) 202001 [hep-th/0501210].

[6] T. DeGrand, R. Hoffmann, S. Schaefer, and Z. Liu, Phys. Rev. D 74 (2006) 054501 [hep-th/0605147].

[7] E. V. Shuryak and J. J. M. Verbaarschot, Nucl. Phys. A 560 (1993) 306 [hep-th/9212088].

[8] J. J. M. Verbaarschot, Phys. Rev. Lett. 72 (1994) 2531 [hep-th/9401059].

[9] P. Damgaard, J. Osborn, D. Toublan, and J. Verbaarschot, Nucl. Phys. B547 (1999) 305 [hep-th/9811212].

[10] J. J. M. Verbaarschot and T. Wettig, Ann. Rev. Nucl. Part. Sci. 50 (2000) 343 [hep-ph/0003017].

[11] P. H. Damgaard, Nucl. Phys. B556 (1999) 327 [hep-th/9903096].

[12] J. J. M. Verbaarschot and T. Wettig, Phys. Rev. D (in press) [arXiv:1407.8393].

[13] M. Creutz, Annals Phys. 322 (2007) 1518 [hep-th/0609187].

[14] M. Creutz, PoS LATTICE 2014 (2014) [arXiv:1410.0883]. 